\title{
A Design Method for Distributed Luenberger Observers
}

\author{
Francisco F. C. Rego ${ }^{12}$, A. Pedro Aguiar ${ }^{3}$, António M. Pascoal ${ }^{2}$ and Colin N. Jones ${ }^{1}$
}

\begin{abstract}
In this paper we address the problem of design of a LTI observer with guaranteed stability, requiring only collective observability, strong connectivity of the communication network, and invertibility of the state transition matrix. We also provide simulation results where the asymptotic performance of the proposed observer is similar to that of time varying distributed Kalman filtering.
\end{abstract}

\section{INTRODUCTION}

\section{A. Motivation}

Spawned by recent advances in wireless sensor networks (WSNs) and distributed sensing, there has been a flurry of activity on the topic of distributed state estimation, see for example [1]-[3] and the references therein. Distributed state estimation and control has a wide range of applications, from network localization to environmental monitoring and formation control of vehicles (see [4]-[6] for an introduction to these topics).

One of the most studied family of distributed estimation algorithms are distributed kalman filters, which extend the theory of Kalman filtering to the distributed setting. Among the many works on this subject are [7]-[15]. We will describe these works in detail in Section III. All of the methods require that the estimation error covariances computed locally are also exchanged among agents, which increases the amount of data needed to communicate. The issue of bandwidth efficiency is of paramount importance in practical applications, since lower bandwidth translates into lower energy consumption and therefore increased operational autonomy. Moreover, since on these methods the estimates have time-varying dynamics, it is difficult to obtain beforehand a convergence rate of the estimation errors.

Those two issues, the need for exchanging covariances and the unknown convergence rate, do not occur for distributed Luenberger observers, also named distributed linear time-invariant (LTI) observers, or distributed fixed gain observers, where the dynamics of the estimation errors is linear time-invariant. Distributed Luenberger observers has been the object of many recent studies [16]-[21], described in detail in Section. However, all of these works have strong assumptions on the kind of dynamical system considered, or the number of communications at each time step. Most of

\footnotetext{
${ }^{1}$ Francisco F. C. Rego and Colin N. Jones are with LA3, STI, EPFL, Lausanne, Switzerland, \{francisco.fernandescastrorego, colin.jones $\}$ depfl.ch

${ }^{2}$ Francisco F. C. Rego and António M. Pascoal are with the Institute for Systems and Robotics (ISR), IST, Univ. Lisbon, Portugal, antoniodisr.ist.utl.pt

3 A. Pedro Aguiar is with the Research Center for Systems and Technologies (SYSTEC) and the Faculty of Engineering of the University of Porto (FEUP), Portugal, pedro.aguiarefe.up.pt
}

those works can only guarantee stability of the observer if the norm of the state transition matrix is below a certain value.

As of the writing of this paper, and to the best of the authors' knowledge a single work [22] provides a LTI distributed observer that guarantees ultimate boundedness of the estimation error for any LTI discrete-time system satisfying only collective observability. However, the method of that paper might suffer from shortcomings in terms of performance as will be mentioned briefly in Section III and will be seen in the Illustrative Example section.

Borrowing from the theory in [10], in this paper we present an alternative design of a distributed Luenberger observer with guaranteed stability, which requires only collective observability, with asymptotic behavior similar to [10], as will be seen in the Illustrative Example section. However, in contrast with the method in [10], since the method in this paper is time-invariant, there is no need for exchanging covariances, which reduces the communication bandwidth burden, and we can obtain an a-priori known convergence rate of the estimation errors.

\section{B. Paper structure}

The paper is structured as follows. The next section, Section II, will describe rigorously the problem at hand and the required assumptions. Section III provides a literature survey on distributed estimation. Next, Section IV describes the estimation algorithm, the design method and the main theorem of this paper, the proof of ultimate boundedness of the estimation errors. In order to illustrate the performance of the algorithm in comparison with other works in the literature Section $\mathrm{V}$ shows the results of the application of the estimation algorithms in an academic illustrative example. Finally, Section VI provides the conclusions of the paper and possible future works.

\section{Notation}

Throughout this paper we will use the symbol $\otimes$ for the Kronecker product. The symbol $\|\cdot\|$ represents the $\mathcal{L}_{2}$ norm. The notation $|\cdot|$ represents the cardinality of a set. The notation $\lfloor\cdot\rfloor$ represents the floor operator, or the rounding down to the closest lower integer, and $\rho(\cdot)$ the spectral radius of a square matrix. $I_{M}$ represents an $M \times M$ identity matrix, and 1 represents a $N \times 1$ vector with ones in every entry. When clear from the context the superscript of a variable, e.g. $x^{i}$, refers to the node index of that variable where $i \in\{1, \ldots, N\}:=\mathcal{N}$. The operator row $(\cdot)$ represents the operator defined by $\operatorname{row}\left(X^{i}\right):=\left[X^{1}, \ldots, X^{N}\right]$, the operator $\operatorname{col}(\cdot)$ represents 
the column operator, i.e. $\operatorname{col}\left(X^{i}\right):=\operatorname{row}\left(X^{i^{T}}\right)^{T}$ and the operator $\operatorname{diag}\left(X^{i}\right)$ results in a block diagonal matrix whose diagonal elements are $X^{1}, \ldots, X^{N}$.

\section{Problem Definition}

Consider the autonomous dynamical system

$$
x_{t+1}=A x_{t}+w_{t}
$$

where $x_{t} \in \mathbb{R}^{n}$ and $w_{t} \in \mathbb{R}^{n}$ denote the state vector and the state noise vector, respectively, at time step $t$, and $A$ is a matrix of appropriate dimensions.

The state vector is observed by a set of sensor nodes $\mathcal{N}$, with cardinality $N=|\mathcal{N}|$. The measurement equation associated to the generic node $i \in \mathcal{N}$ is defined as

$$
y_{t}^{i}=C^{i} x_{t}+v_{t}^{i}
$$

where $y_{t}^{i} \in \mathbb{R}^{m_{i}}$ and $v_{t}^{i} \in \mathbb{R}^{m_{i}}$ denote the observation vector and the observation noise vector, respectively, considered at time $t$, and $C^{i}$ is a matrix of appropriate dimensions. The overall network can be described by the pair $(\mathcal{N}, \mathcal{A})$ where $\mathcal{A} \subseteq \mathcal{N} \times \mathcal{N}$ is the set of node pairs denoting the directed connections between the nodes. We denote by $\mathcal{N}^{i}$ the set of in-neighbors of $i$, i.e., $\mathcal{N}^{i}:=\{j:(j, i) \in \mathcal{A}\}$. Some basic assumptions on observability of the system and intensity of the disturbances follow.

Assumption A1 The system is collectively observable, i.e. the pair $(A, C)$ is observable where $C:=\operatorname{col}\left(C^{i}\right)$.

Note that we only assume global observability and not local observability, i.e. we do not assume that the pair $\left(A, C^{i}\right)$ is observable for any $i \in \mathcal{N}$.

Assumption A2 The dynamics matrix $A$ is invertible.

Remark As is shown in the appendix B of [22], assumption A2 is mild since we can design a stable estimator by only estimating the modes of the state associated with the nonzero eigenvalues, since all the other modes will vanish over time.

Since in this paper we aim to guarantee ultimate boundedness of the estimation error at every node we require the following assumption on the magnitude of the disturbances

Assumption A3 The $\mathcal{L}_{2}$ norm of the disturbances satisfy

$$
\left\|w_{t}\right\| \leq \epsilon_{w}, \quad\left\|v_{t}^{i}\right\| \leq \epsilon_{v^{i}}, i \in \mathcal{N},
$$

for some constants $\epsilon_{w}>0$ and $\epsilon_{v^{i}}>0$.

The following assumption describes the communication limitations among sensors.

Assumption A4 At each time step the nodes are allowed to communicate once according to the network structure defined by $\mathcal{A}$.

In this paper we consider a matrix $\Pi$ whose component $(i, j)$ is equal to $\pi^{i, j}$, where $\pi^{i, j}=0$ if $(i, j) \notin \mathcal{A}$, which is termed the consensus matrix and is assumed to satisfy the following standard assumptions:
Assumption A5 The consensus matrix $\Pi$ is doubly stochastic and primitive ${ }^{1}$.

Remark Note that if the graph is bidirectional, i.e. if $(i, j) \in \mathcal{A}$ implies $(j, i) \in \mathcal{A}$, then Assumption A5 can be satisfied by designing $\Pi$ with Metropolis local-degree weights [23].

It can be shown (Theorem 3 of [24]) that it is possible to compute a consensus matrix satisfying A5 if and only if the network is strongly connected, i.e. for every two nodes $i$ and $j$ there is a path from $i$ to $j$ and from $j$ to $i$.

If the network is not strongly connected, it is still possible to use the results of this paper to design a distributed estimator, using some of the methods in [22], given that all source components, strongly connected subsets of the network with no incoming edges from the rest of the network, are collectively observable.

Considering assumptions A1-A5 this paper addresses the problem of distributed state estimation, i.e. each node reconstructs locally the state of the global system (1), as $\hat{x}_{t}^{i}$, with estimation error, $x_{t}-\hat{x}_{t}^{i}$, converging to an ultimate bound proportional to the magnitude of the disturbances, $\epsilon_{w}>0$ and $\epsilon_{v^{i}}, i \in \mathcal{N}$.

\section{RELATED WORKS}

In what follows we give a very brief survey of the state of the art in distributed estimation. The reader is referred to [2] for a recent literature survey of this subject and to [1] for an overview of the technical details associated with consensus-based distributed estimation.

\section{A. Distributed Kalman filtering}

One of the standard methods in state estimation for linear systems is Kalman filtering, where the covariance of the state estimation error is computed at each time and the observer gain is computed based on that covariance. In the distributed setting, in general, the computation of an approximation of the estimation error covariance at each node involves the communication of the covariances among nodes.

A seminal work in distributed Kalman filtering is [7]. The key technique used involves writing the Kalman filter equations in information form, i.e. in terms of the inverse of the covariance matrix, termed the information matrix. The latter is then computed as the average of local information matrices and the global measurement is computed as the averaging of linear transformations of the local measurements.

A further development in distributed Kalman filter appears in [8], where it is shown how to compute the optimal gain matrix of a linear observer given that at each time an agent communicates with a limited set of neighbours. However, it is also shown that the computation of this optimal gain requires data from the neighbours of the neighbours,

\footnotetext{
${ }^{1}$ A doubly stochastic matrix is a square matrix of nonnegative real numbers, whose rows and columns sum to 1 . A primitive matrix is a nonnegative square matrix $A$ such that there exists a positive integer $k$ such that all elements of $A^{k}$ are strictly positive
} 
which requires two communication cycles. In addition the work in [8] provides a simplified, more tractable, version the distributed kalman filter with stability guarantees, but which requires local observability, i.e. the state of the system can be observed by each sensor individually. The work in [9] builds on the results in [8] to tackle the problem of simultaneous state estimation and tracking.

Strong theoretical guarantees on stability of the distributed consensus based Kalman filter, without requiring local observability or an infinite number of communications at each step, are available in [10] the authors summarize the different consensus-based approximations of a Kalman filter in a distributed setting: consensus on information, consensus on measurements, and an hybrid version. The work [10] also provides a stability proof of the hybrid version of the distributed Kalman filter given only local observability. An extension of this work to the case of the extended Kalman filter is given in [11], [12]. Some of the technical details of the Theorems in [11] are provided in [25].

A different perspective on distributed Kalman filtering, which makes use of distributed matrix inversion algorithms, is given in [13], [14], where the authors describe a version of the distributed Kalman filter in the consensus on measurements form, as described in [10], where the computations are scalable with the number of nodes. This is achieved by estimating only local states at each node, and not the full state. Furthermore, the authors discuss how to take advantage of the sparseness of the dynamics and observation matrices, so that only local communications are required. For this purpose they propose a distributed matrix inversion algorithm, the distributed iterate collapse inversion (DICI), for covariance inversion, assuming that the dynamics matrix is a banded matrix.

Distributed Kalman filtering can be cast as fusion of several estimators at each time step using a particular fusion method. A survey on different fusion rules which provide stable observers is given in [15], which provides a state fusion approach to distributed Kalman filtering instead of a consensus-based approach. The paper also derives a stability condition which guarantees that the covariance of the estimates is uniformly upper bounded on all the sensor nodes where the estimator is running. Two fusion methods (denoted covariance and ellipsoidal intersection) that satisfy the stability condition are proposed.

It is worth mentioning that all the mentioned methods in this sub-subsection involve, in one form or another, transmitting the covariance of the estimation error at each node. In order to avoid communicating covariances among nodes, to save bandwidth, one could use distributed LTI observers, or distributed Luenberger observers.

\section{B. LTI Observers}

The concept of distributed Luenberger observers is developed in [16], which studies the network tracking capacity of distributed Luenberger observers, i.e., distributed estimators with fixed innovation gain, which communicate only once between agents between measurements. Given a set of sensors and a communication network among them, the network tracking capacity is the maximum vector induced 2 - norm of the dynamics matrix of an observed system such that it is possible to compute a fixed gain matrix for a distributed observer with guaranteed ultimate boundedness of the estimation error. The authors study observers which depend only on one parameter to provide an analytical lower bound on the network tracking capacity, and show that this lower bound is always greater than one for strongly connected communication graphs. On the other hand, when multiple iterations are allowed between measurements, in [17] the authors show that it is possible to design distributed estimators with fixed innovation gain, which are stable if enough iterations of the consensus algorithm are performed. Namely, if the number of iterations are greater or equal to the primitivity index of the consensus matrix.

Following the same framework of [16] the authors in [18]-[21] provide variations of the distributed Kalman filter algorithms, with consensus on pseudo-innovations, and analyze their stability. One of the main assumptions on those papers is that the 2-norm of the dynamics matrix of the system dynamics is smaller or equal to the network tracking capacity defined in [16].

In contrast to the previously mentioned works in distributed LTI observers which assume a bound on the 2norm of the state transition matrix the work in [22] provides a method of designing stable distributed LTI observers with very mild assumptions, which are weaker than strong connectivity of the network. Specifically it is only required that all source components, strongly connected subsets of the network with no incoming edges from the rest of the network, are collectively observable. Since in that paper it is proposed that the gains of all the observers except one are chosen randomly, it is apparent that this method might not be competitive in terms of performance, i.e. convergence rate and ultimate estimation error bound.

In order to design a distributed LTI observer which matches as close as possible the asymptotic performance of a distributed Kalman filter, in this paper we follow the estimation method and the theory in [10]. To obtain a distributed LTI observer from the method in [10], we provide a method of computing time invariant information matrices to each node that guarantee stability of the observer.

\section{MAIN Result}

\section{A. Algorithm}

In what follows, to simplify the notation, we will omit the time index $t$. For this purpose, for a time-varying vector $x_{t}$, when omitting the time index $t$ we will use the notation $x^{+}$to refer to $x_{t+1}$.

The algorithm proposed in this paper has the following form:

$$
\hat{x}^{i+}=A\left(\Omega^{i}\right)^{-1}\left(\sum_{j \in \mathcal{N}} \pi^{i, j} \bar{\Omega}^{i} \hat{x}^{j}+C^{i T}\left(R^{i}\right)^{-1} y^{i}\right),
$$


where $\Omega^{i}$, and $\bar{\Omega}^{i}$ are appropriately chosen positive definite matrices of size $n \times n$, where $n$ is the size of the state $x$, and $R^{i}$ is a positive definite matrix of size $m^{i} \times m^{i}$, where $m^{i}$ is the size of the measurement $y^{i}$.

This observer algorithm is similar to the distributed Kalman filter with consensus on information given in [10]. However, in this work we consider that the matrices $\Omega^{i}$, and $\bar{\Omega}^{i}$ are fixed in time.

To follow closely the principles of Kalman filtering, if $v^{i}$ is a zero mean Gaussian noise with known covariance, the matrix $R^{i}$ should be set equal to the covariance of $v^{i}$.

The main problem that we address in this paper is how to compute matrices $\Omega^{i}$, and $\bar{\Omega}^{i}$. The design method is described in the next subsection.

\section{B. Design}

The design process is as follows. We first choose the parameter $0<\tilde{\beta}<1$ and define the matrix

$$
S i:=C^{i T}\left(R^{i}\right)^{-1} C^{i},
$$

With these two parameters we compute the matrix

$$
\tilde{\Omega}^{i}:=\sum_{\tau=0}^{\bar{k}-1} \tilde{\beta}^{\tau}\left(A^{-\tau}\right)\left(\sum_{j \in \mathcal{N}} \pi_{\tau}^{i, j} S^{j}\right) A^{-\tau},
$$

where $\pi_{\tau}^{i, j}$ is the element $i, j$ of the matrix $\Pi^{\tau}$, and $\bar{k}:=$ $k+n$ where $k$ is the primitivity index of $\Pi$, i.e. the lowest integer such that $\Pi^{k}$ is strictly positive, and $n$ is the dimension of the state. It can be seen that, from collective observability and the fact that $\Pi^{k}$ is strictly positive, $\tilde{\Omega}^{i}$ is positive definite

Then we may compute matrix $\bar{\Omega}^{i}$ as

$$
\bar{\Omega}^{i}:=\tilde{\beta}\left(A^{-1}\right) \tilde{\Omega}^{i} A^{-1}
$$

Finally we can compute $\Omega^{i}$ as follows

$$
\Omega^{i}:=S^{i}+\sum_{j \in \mathcal{N}} \pi^{i, j} \bar{\Omega}^{j}
$$

The motivation for this choice of matrices $\Omega^{i}$, and $\bar{\Omega}^{i}$ is given in the proof of stability presented in the next subsection.

\section{Main Theorem}

We now present the main result of this paper.

Theorem 1 Consider the distributed LTI observer (3), with matrices $\Omega^{i}$, and $\bar{\Omega}^{i}$ computed as in (4)-(5). Then, given assumptions A1-A5, the estimation errors $\hat{x}^{i}-x, i \in \mathcal{N}$ are ultimately bounded with ultimate bounds on $\left\|\hat{x}^{i}-x\right\|, i \in \mathcal{N}$ proportional to the bounds on the magnitude of the noise $\epsilon_{w}>0$ and $\epsilon_{v^{i}}, i \in \mathcal{N}$.

Proof 1 We first consider the noiseless case

$$
\begin{aligned}
x^{+} & =A x \\
y^{i} & =C^{i} x
\end{aligned}
$$

Defining the estimation error as $\eta^{i}:=\hat{x}^{i}-x$ one has from (3) that

$$
\begin{aligned}
\eta^{i+} & =A\left(\Omega^{i}\right)^{-1}\left(\sum_{j \in \mathcal{N}} \pi^{i, j} \bar{\Omega}^{i} \hat{x}^{j}+S^{i} x-\Omega^{i} x\right) \\
& =A\left(\Omega^{i}\right)^{-1}\left(\sum_{j \in \mathcal{N}} \pi^{i, j} \bar{\Omega}^{i} \eta^{j}\right),
\end{aligned}
$$

where the last equality comes from (5). We can then establish the following relation.

$$
\begin{aligned}
\Omega^{i} & =S^{i}+\sum_{j \in \mathcal{N}} \pi^{i, j} \bar{\Omega}^{j} \\
& =S^{i}+\sum_{\tau=1}^{\bar{k}} \tilde{\beta}^{\tau}\left(A^{-\tau}\right)\left(\sum_{j \in \mathcal{N}} \pi_{\tau}^{i, j} S^{j}\right) A^{-\tau} \\
& =\tilde{\Omega}^{i}+\tilde{\beta}^{\bar{k}}\left(A^{-\bar{k}}\right)\left(\sum_{j \in \mathcal{N}} \pi_{\bar{k}}^{i, j} S^{j}\right) A^{-\bar{k}} \\
& \geq \tilde{\Omega}^{i}
\end{aligned}
$$

where, on the last inequality, we used the fact that the system is collectively observable and $\pi_{\bar{k}}^{i, j}>0$ for all $i, j \in \mathcal{N}$, and thus $\tilde{\beta}^{\bar{k}}\left(A^{-\bar{k}}\right)\left(\sum_{j \in \mathcal{N}} \pi_{\bar{k}}^{i, j} S^{j}\right) A^{-\bar{k}}$ is positive definite.

Defining the local cost as $\mathcal{L}^{i}:=\eta^{i T} \bar{\Omega}^{i} \eta^{i}$ one has, using the fact that $\Omega^{i} \geq \tilde{\Omega}^{i}$ and (4)-(5), and using Lemma 2 of [25]

$$
\begin{aligned}
& \mathcal{L}^{i+}= \\
& =\tilde{\beta}\left(\sum_{j \in \mathcal{N}} \pi^{i, j} \bar{\Omega}^{i} \eta^{j}\right)^{T}\left(\Omega^{i}\right)^{-1} \tilde{\Omega}^{i}\left(\Omega^{i}\right)^{-1}\left(\sum_{j \in \mathcal{N}} \pi^{i, j} \bar{\Omega}^{i} \eta^{j}\right) \\
& \leq \tilde{\beta}\left(\sum_{j \in \mathcal{N}} \pi^{i, j} \bar{\Omega}^{i} \eta^{j}\right)^{T}\left(\Omega^{i}\right)^{-1} \Omega^{i}\left(\Omega^{i}\right)^{-1}\left(\sum_{j \in \mathcal{N}} \pi^{i, j} \bar{\Omega}^{i} \eta^{j}\right) \\
& \leq \tilde{\beta}\left(\sum_{j \in \mathcal{N}} \pi^{i, j} \bar{\Omega}^{i} \eta^{j}\right)^{T}\left(\sum_{j \in \mathcal{N}} \pi^{i, j} \bar{\Omega}^{j}\right)^{-1}\left(\sum_{j \in \mathcal{N}} \pi^{i, j} \bar{\Omega}^{i} \eta^{j}\right) \\
& \leq \tilde{\beta} \sum_{j \in \mathcal{N}} \pi^{i, j} \eta^{j T} \bar{\Omega}^{i} \eta^{j}=\tilde{\beta} \sum_{j \in \mathcal{N}} \pi^{i, j} \eta^{j T} \bar{\Omega}^{i} \eta^{j} .
\end{aligned}
$$

In vector form, defining $\mathcal{L}:=\operatorname{col}\left(\mathcal{L}^{i}\right)$ one has

$$
\mathcal{L}^{+} \leq \tilde{\beta} \Pi \mathcal{L},
$$

where the inequality is interpreted element-wise.

Finally, defining the Lyapunov function

$$
\mathcal{V}:=\sum_{i \in \mathcal{N}} \mathcal{L}^{i}=\mathbf{1}^{T} \mathcal{L}
$$

one has

$$
\mathcal{V}^{+}=\mathbf{1}^{T} \mathcal{L}^{+} \leq \tilde{\beta} \mathbf{1}^{T} \Pi \mathcal{L}=\tilde{\beta} \mathbf{1}^{T} \mathcal{L}=\tilde{\beta} \mathcal{V},
$$


Since the Lyapunov function decreases at each step, we have that the estimation errors for the noiseless case converge to zero.

Since for the noiseless case the estimation errors converge asymptotically to zero, from classical results on LTI systems the Theorem follows.

\section{ILlustrative EXAMPle}

In this section we will test the performance of the algorithm proposed in this paper on an academic example and compare to other methods in the literature, namely the scalar gain observer in [16], illustrating the performance of an algorithm that requires a bound on the $\mathcal{L}_{2}$ norm of the state transition matrix, the distributed Kalman filter with consensus on information (and not on measurements) algorithm in [10], and the method in [22]. In the algorithm proposed in this paper the parameter choice for $\tilde{\beta}$ was 0.7 .

We will consider a network of 11 nodes. The dynamical system considered is the following. The state transition matrix is defined as $A:=\operatorname{diag}\left(A^{i}\right)$ with

$$
A^{i}:=\lambda\left[\begin{array}{cc}
\cos \left(\phi_{i}\right) & -\sin \left(\phi_{i}\right) \\
\sin \left(\phi_{i}\right) & \cos \left(\phi_{i}\right)
\end{array}\right]
$$

for each $i \in \mathcal{N}$, where $\phi_{i}$ ranges uniformly from $0.01 \frac{\pi}{2}$ at $i=1$ to $0.1 \frac{\pi}{2}$ at $i=N$ and $\lambda$ will be defined later.

Defining the basis vector $e^{i}$ which is a row vector with 1 at position $i$ and zero at every other position, the observation matrices are defined as

$$
C^{i}:=\left[\begin{array}{c}
e^{i T}-e^{(i+1) T} \\
e^{(i-1) T}-e^{i T}
\end{array}\right] \otimes I_{2},
$$

except at $i=1$, where we replace $i-1$ by $N$, and $i=N$, where we define $C^{N}:=e^{N T} \otimes I_{2}$. It can be observed that from this choice of state transition and observation matrices we have collective observability but not local observability at each node, thus we require distributed observers to reconstruct the state.

The process and measurement noises are generated randomly with a Gaussian distribution. The covariances of the noises were $Q=100 I_{2 N}$ and $R^{i}=10^{-4} I_{m_{i}}$. The initial state is also randomly generated with a Gaussian distribution with covariance $P_{0}=10^{10} I_{2 N}$.

The communication network considered was an undirected circular network, i.e. the neighbor set at each node is defined as $\mathcal{N}^{i}:=\{i-1, i+1\}$ except at node $i=1$ where it is $\mathcal{N}^{i}:=\{N, 2\}$, and at node $i=N$ where it is $\mathcal{N}^{i}:=\{N-1,1\}$.

In what follow we will compare the different algorithms in terms of the norm of the global estimation, i.e. of $\left\|\operatorname{col}\left(\hat{x}^{i}-x\right)\right\|$. To remove the randomness effect of a single simulation run, we perform 50 runs and plot the average. In the following plots we will present the results of the scalar gain observer in [16] in red, the distributed Kalman filter in [10] in green, the method in [22] in blue, and the algorithm of this paper in magenta.

For reference we also plot the norm of the stacked state vector $\left\|\mathbf{1} \otimes I_{2 N} x\right\|=\sqrt{N}\|x\|$ in black.
The results for $\lambda=0.9$ are shown in Figure 1 and a zoom of this plot is shown in Figure 2 and the results for $\lambda=1.05$ are shown in Figure 3.

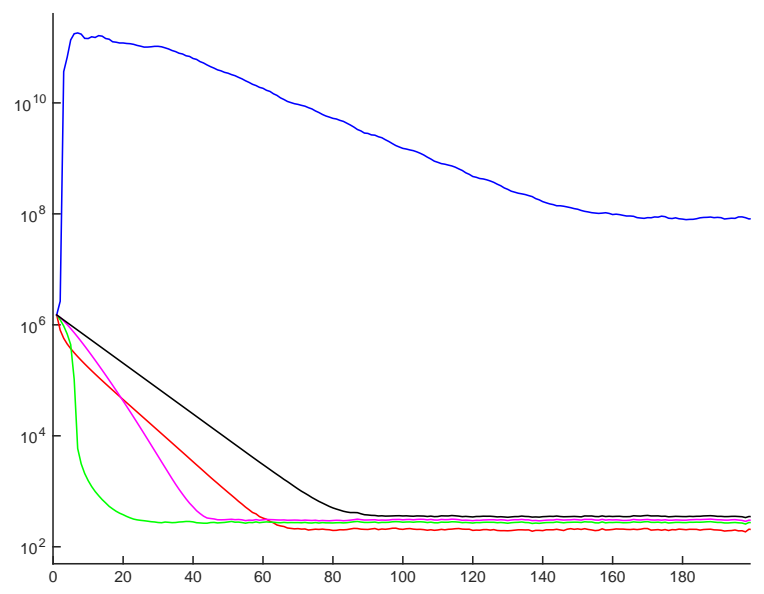

Fig. 1. Norms of global estimation errors for $\lambda=0.9$

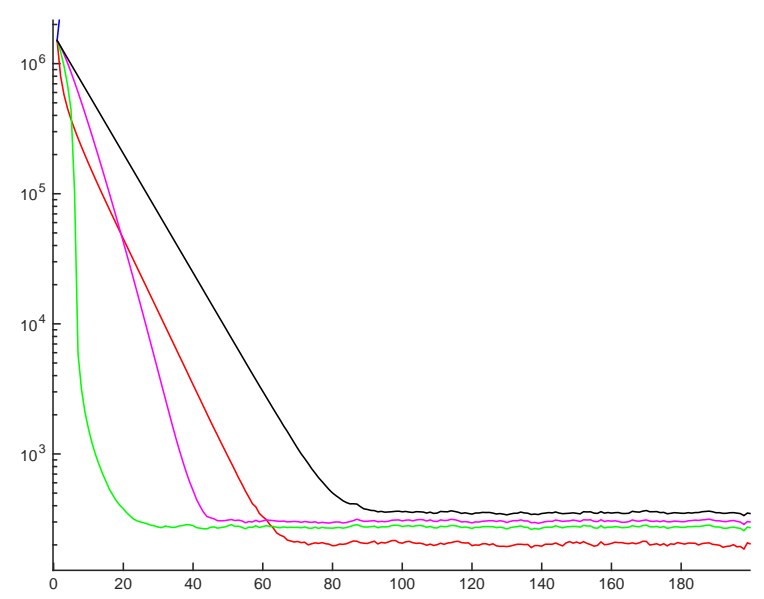

Fig. 2. Zoom of Figure 1 showing ultimate bounds of estimation error

From Figures 1 and 2 one can observe that all estimators remain stable. However, the observer from [22] has worse convergence rates and worse ultimate boundedness than the state. This is not surprising since the observer gains on all the nodes except one are assigned randomly. We can also note that the ultimate bound of the estimation error of the observer proposed in this paper is worse but close to the ultimate bound observed for the method in [10]. Figure 2 also shows a ultimate lower ultimate bound for the algorithm in [16] possibly due to the fact that in [16], unlike the other works considered here, the measurements $y^{i}$ are exchanged at each time.

From Figure 3 one can see that since the norm of the state transition matrix is greater than the bound required for stability on [16] the method in that paper is unstable for this case, although with an increase rate lower than that of the state. Also, one can observe that, as expected, the 


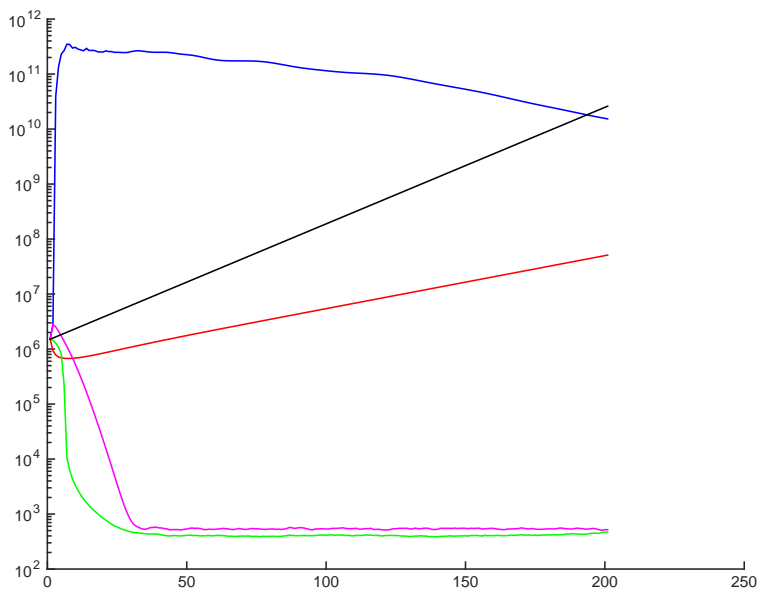

Fig. 3. Norms of global estimation errors for $\lambda=1.05$

observer designed with the method in [22] is stable, but has a very low convergence rate when compared to the other stable methods. Finally one can see that the asymptotic performance of the method of this paper is comparable to that of the method in [10].

\section{CONCLUSION}

In this paper we provided a design of a LTI observer with guaranteed stability, which only requires collective observability, strong connectivity of the communication network, and invertibility of the dynamics matrix. On simulation results of an illustrative example it was observed that the asymptotic performance is similar to that of [10].

Following up on this work, possible future topics of research include optimizing the selection of the parameter $\tilde{\beta}$, design plug and play procedures for adding and removing sensors, since we have a-priori known convergence rates one can consider using progressive quantizers to exchange messages as in [26], or derive other design methods of distributed LTI observers.

\section{ACKNOWLEDGMENTS}

The work of the third author was supported by the EC Project WiMUST (GA No. 645141).

\section{REFERENCES}

[1] F. Garin and L. Schenato, "A survey on distributed estimation and control applications using linear consensus algorithms," in Networked Control Systems. Springer, 2010, pp. 75-107.

[2] W. Li, Z. Wang, G. Wei, L. Ma, J. Hu, and D. Ding, "A survey on multisensor fusion and consensus filtering for sensor networks," Discrete Dynamics in Nature and Society, vol. 2015, 2015.

[3] P. Rawat, K. D. Singh, H. Chaouchi, and J. M. Bonnin, "Wireless sensor networks: a survey on recent developments and potential synergies," The Journal of supercomputing, vol. 68, no. 1, pp. 1-48, 2014.

[4] C. Soares, J. Xavier, and J. Gomes, "Simple and fast convex relaxation method for cooperative localization in sensor networks using range measurements," Signal Processing, IEEE Transactions on, vol. 63, no. 17, pp. 4532-4543, 2015.
[5] K. Aberer, S. Sathe, D. Chakraborty, A. Martinoli, G. Barrenetxea, B. Faltings, and L. Thiele, "Opensense: open community driven sensing of environment," in Proceedings of the ACM SIGSPATIAL International Workshop on GeoStreaming. ACM, 2010, pp. 39-42.

[6] R. Ghabcheloo, A. P. Aguiar, A. Pascoal, C. Silvestre, I. Kaminer, and J. Hespanha, "Coordinated path-following in the presence of communication losses and time delays," SIAM Journal on Control and Optimization, vol. 48, no. 1, pp. 234-265, 2009.

[7] R. Olfati-Saber, "Distributed kalman filter with embedded consensus filters," in Decision and Control, 2005 and 2005 European Control Conference. CDC-ECC'05. 44th IEEE Conference on. IEEE, 2005, pp. 8179-8184.

[8] —, "Kalman-consensus filter : Optimality, stability, and performance," in Decision and Control, 2009 held jointly with the 2009 28th Chinese Control Conference. CDC/CCC 2009. Proceedings of the 48th IEEE Conference on, Dec 2009, pp. 7036-7042.

[9] R. Olfati-Saber and P. Jalalkamali, "Coupled distributed estimation and control for mobile sensor networks," Automatic Control, IEEE Transactions on, vol. 57, no. 10, pp. 2609-2614, 2012.

[10] G. Battistelli, L. Chisci, G. Mugnai, A. Farina, and A. Graziano, "Consensus-based linear and nonlinear filtering," Automatic Control, IEEE Transactions on, 2014.

[11] G. Battistelli and L. Chisci, "Stability of consensus extended kalman filtering for distributed state estimation," in World Congress, vol. 19, 2014, pp. 5520-5525.

[12] — , "Stability of consensus extended kalman filter for distributed state estimation," Automatica, vol. 68, pp. 169-178, 2016.

[13] U. A. Khan and J. M. Moura, "Distributed kalman filters in sensor networks: Bipartite fusion graphs," in 2007 IEEE/SP 14th Workshop on Statistical Signal Processing. IEEE, 2007, pp. 700-704.

[14] — , "Distributing the kalman filter for large-scale systems," Signal Processing, IEEE Transactions on, vol. 56, no. 10, pp. 4919-4935, 2008

[15] J. Sijs and P. van den Bosch, "Heterogeneous state estimation in dynamic networked systems," Control Theory \& Applications, IET, vol. 9, no. 15, pp. 2232-2241, 2015.

[16] U. A. Khan, S. Kar, A. Jadbabaie, and J. M. Moura, "On connectivity, observability, and stability in distributed estimation," in Decision and Control (CDC), 2010 49th IEEE Conference on. IEEE, 2010, pp. 6639-6644.

[17] U. A. Khan and A. Jadbabaie, "On the stability and optimality of distributed kalman filters with finite-time data fusion," in American Control Conference (ACC), 2011. IEEE, 2011, pp. 3405-3410.

[18] S. Das and J. M. Moura, "Distributed state estimation in multi-agent networks," in 2013 IEEE International Conference on Acoustics, Speech and Signal Processing. IEEE, 2013, pp. 4246-4250.

[19] — - "Distributed linear estimation of dynamic random fields," in Communication, Control, and Computing (Allerton), 2013 51st Annual Allerton Conference on. IEEE, 2013, pp. 1120-1125.

[20] — - "Distributed kalman filtering," in 21st European Signal Processing Conference (EUSIPCO 2013), 2013.

[21] — - "Distributed kalman filtering with dynamic observations consensus," IEEE Transactions on Signal Processing, vol. 63, no. 17, pp. 4458-4473, 2015.

[22] S. Park and N. C. Martins, "Design of distributed lti observers for state omniscience," IEEE Transactions on Automatic Control, 2016.

[23] L. Xiao and S. Boyd, "Fast linear iterations for distributed averaging," Systems \& Control Letters, vol. 53, no. 1, pp. 65-78, 2004.

[24] D. J. Hartfiel, "Concerning diagonal similarity of irreducible matrices," Proceedings of the American Mathematical Society, vol. 30, no. 3, p. 419, 1971. [Online]. Available: http://www.jstor.org/stable/2037708?origin=crossref

[25] G. Battistelli and L. Chisci, "Kullback-leibler average, consensus on probability densities, and distributed state estimation with guaranteed stability," Automatica, vol. 50, no. 3, pp. 707 - 718, 2014.

[26] F. F. C. Rego, Y. Pu, A. Alessandretti, A. P. Aguiar, A. M. Pascoal, and C. N. Jones, "Design of a distributed quantized luenberger filter for bounded noise," in 2016 American Control Conference (ACC), July 2016, pp. 6393-6398. 